\title{
Ecological potential datasets (Ecopot)
}

Maxime Sweetlove, Jeroen Van Wichelen, Elie Verleyen \& Wim Vyverman
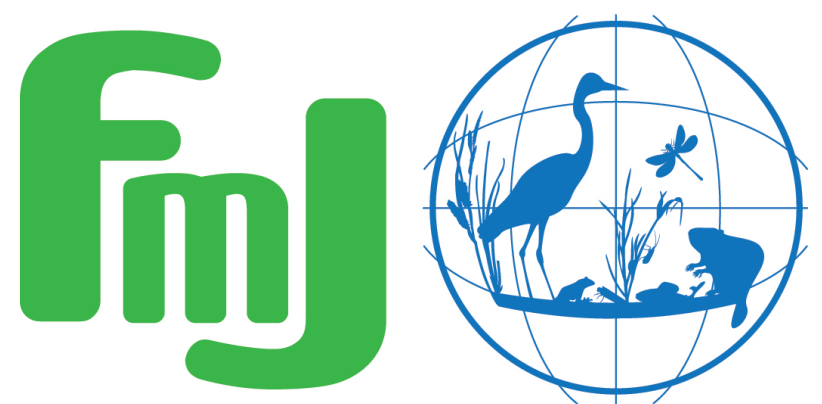

Freshwater Metadata Journal 



\title{
Ecological potential datasets (Ecopot)
}

\author{
Maxime Sweetlove ${ }^{1,3}$, Jeroen Van Wichelen ${ }^{1,2}$, Elie Verleyen ${ }^{1} \&$ Wim Vyverman ${ }^{1}$ \\ 1 Ghent University, Ghent, Belgium \\ 2 Instituut voor Natuur- en Bosonderzoek, Brussels, Belgium \\ 3 Royal Belgian Institute for Natural Sciences, Brussels, Belgium
}

Please cite this paper as follows: Sweetlove, M., Van Wichelen, J., Verleyen, E. \& Vyverman, W., 2018. Ecological potential datasets (Ecopot). Freshwater Metadata Journal 38: 1-4.

https://doi.org/10.15504/fmj.2018.38

Received: 2018-10-16 / Published: 2018-10-18

\section{Keywords}

phytoplankton

\section{Short description of the dataset/summary}

Monitoring data from the Ecopot projects: Determining the maximal and good ecological potential, as well as the current state of Flemish regional water bodies. The data contains phytoplankton counts of Flemish lakes and pools.

\section{General information}

dataset entry ID: $\quad$ SF_4

\section{name of the dataset:}

full name of the dataset: $\quad$ Ecopot

dataset short name: Ecopot

type of dataset: species distribution data

data type: point data/observation data

science keywords according to GCMD:

topic:

Biosphere, Biological Classification, Terrestrial Hydrosphere

ISO topic category according to ISO 19115:

Biota, Environment, Inland Waters

\section{INSPIRE keywords according to GEMET:}

Habitats and biotopes, Species distribution

own science keywords:

related project:

phytoplankton, pool, lake, Flanders

funding:

VMM.AMO.KRW.BLOKK

INBO, VMM 


\section{Technical and administrative specifications}

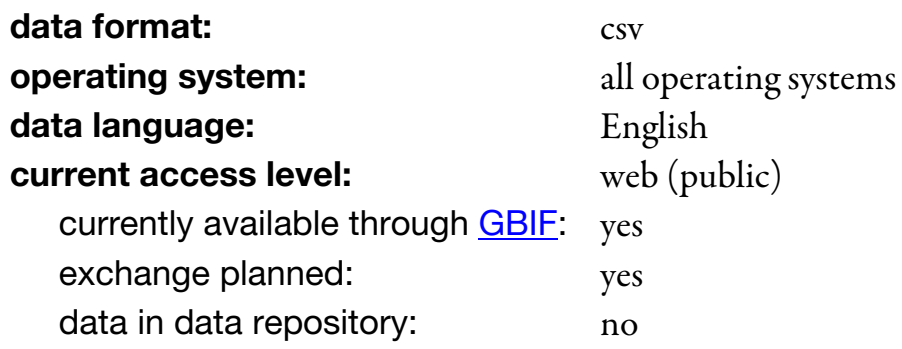

Do you plan to publish the data on the Freshwater Biodiversity Data Portal:

$\begin{array}{ll}\begin{array}{l}\text { update level: } \\ \text { documentation: } \\ \text { type: }\end{array} & \begin{array}{l}\text { completed } \\ \text { contact details: }\end{array} \\ \text { metadata contact person: } & \\ \text { first, last name: } & \text { Jeroen Van Wichelen } \\ \text { phone: } & +32025250277 \\ \text { email: } & \text { jeroen.vanwichelen@inbo.be } \\ \text { institution: } & \text { Instituut natuur- en bosonderzoek (INBO) } \\ \text { address: } & \text { Kliniekstraat } 25 \\ \text { postal code, city: } & 1070 \text { Anderlecht } \\ \text { country } & \text { Belgium } \\ \text { web address: } & \text { https://www.inbo.be/en } \\ \text { technical contact person: } & \\ \text { first, last name: } & \text { Jeroen Van Wichelen } \\ \text { phone: } & +32025250277 \\ \text { email: } & \text { jeroen.vanwichelen@inbo.be } \\ \text { scientific contact person: } & \\ \text { first, last name: } & \text { Jeroen Van Wichelen } \\ \text { phone: } & +32025250277 \\ \text { email: } & \text { jeroen.vanwichelen@inbo.be }\end{array}$

\section{Intellectual property rights and citation}

dataset creator (data compiler):

contact name:

Wim Vyverman

contact email:

wim.vyverman@ugent.be

contact institution:

Ghent University

data contributors to/owners of this dataset:

multiple

number:

3

\section{data contributor/owner 1 :}

contact name:

Wim Vyverman

contact email:

contact institute:

wim.vyverman@ugent.be

Ghent University

criteria for using this part of the dataset:

The dataset is publicly available (data portal, data archive) and can be used without restrictions, but dataset creator/data contributors must be informed 


\section{data contributor/owner 2:}

contact name:

contact email:

contact institute: prior to publication. Data must be acknowledged and cited correctly.

Luc Denys

luc.denys@inbo.be

Instituut natuur- en bosonderzoek

criteria for using this part of the dataset:

The dataset is publicly available (data portal, data archive) and can be used without restrictions, but dataset creator/data contributors must be informed prior to publication. Data must be acknowledged and cited correctly.

data contributor/owner 3:

contact name:

Jeroen Vanwichelen

contact email:

jeroen.vanwichelen@inbo.be

contact institute:

Ghent University

criteria for using this part of the dataset:

The dataset is publicly available (data portal, data archive) and can be used without restrictions, but dataset creator/data contributors must be informed prior to publication. Data must be acknowledged and cited correctly.

citation of this dataset:

author(s):

Louette, G., Van Wichelen, J., Packet, J., Warmoes, T., Vyverman, W., Denys, L., Van Damme, S., Van Colen, W., Schoelynck, J., Muylaert, K., Meire, P., De Smedt, S., Lock, K., Simoens, I., Van Looy, K., Leyssen, A., Pals, A. \& van Dam, $\mathrm{H}$.

title and journal (name, number, pages):

Bepalen van het maximaal en het goed ecologisch potentieel, alsook de huidige toestand voor de zeventien Vlaamse (gewestelijke) waterlischamen die verglijkbaar zijn met de categorie meren

year:

2008

doi:

https://doi.org/10.15468/3pxfgi

\section{citation of the metadata:}

author(s):

Sweetlove M., Van Wichelen J., Verleyen E. \& Vyverman W.

title and journal (name, number, pages):

Ecological potential datasets (Ecopot). Freshwater Metadata Journal 38: 1-4

year:

2018

doi:

https://doi.org/10.15504/fmj.2018.38

comments:

There are two datasets published:

https://doi.org/10.15468/3pxfgi

https://doi.org/10.15468/jz1cdj

\section{General data specifications}

regional coverage of the dataset:

spatial extent of the dataset:

continents:

regional

countries:

Europe

Europe: Belgium

world climatic regions according to Köppen:

European ecoregions according to Illies (WFD):

Group C: temperate/mesothermal climates

ecosystem type:

Western Plains (ER13)

lakes/ponds 
covered timeframe:

$2006-2008$

\section{Site specifications}

coordinate system/grid data:

grid data available:

site coding available:

number of sites:

\section{Biological data}

biological data origin:

from sampling,

Ecopot projects: VMM.AMOKRW.GROVMECH;

VMM.AMO.KRW.SCHULENS; VMM.AMO.KRW.VINNE;

VMM.AMO.KRW.BLOKK; VMM.AMO.KRW.HAZ;

VMM.AMO.KRW.ECOPOT-Meren; VMM.AMO.KRW.GAV

organism group addressed: latitude/longitude, format: DD

no

no

$<100$ phytoplankton

\section{Sample resolution}

phytoplankton:

taxonomic resolution:

level:

genus

taxonomic coding:

taxalist according to:

no reference taxalist used

coding system:

no coding

\section{Other specifications}

GIS layers, shape files related to the dataset:

$\begin{array}{ll} & \text { no data available } \\ \text { availability of photos: } & \text { no } \\ \text { availability of maps: } & \text { no }\end{array}$

quality control procedures:

Were any quality control procedures applied to your dataset?

no

\section{Acknowledgements}

The authors thank the Belgian Science Policy (Belspo) for funding the SAFRED project (Saving freshwater biodiversity research data) that allowed processing and publishing these data. 